\title{
Fraturas causadas por armas de fogo: Epidemiologia e taxa de infecção*
}

\section{Firearm-Related Fractures: Epidemiology and Infection Rate}

\author{
Daniel Baumfeld ${ }^{1}$ Auro Sérgio Perdigão de Brito ${ }^{1}$ Maíra Soares Torres ${ }^{1}$ Kassio Lohner Prado ${ }^{1}$ \\ Marco Antonio Percope de Andrade ${ }^{1}$ Tulio Vinicius de Oliveira Campos ${ }^{1}$ \\ ${ }^{1}$ Departamento do Aparelho Locomotor, Universidade Federal de \\ Minas Gerais, Belo Horizonte, Brasil \\ Rev Bras Ortop 2020;55(5):625-628. \\ Endereço para correspondência Daniel Baumfeld, MD, PhD, \\ Departamento do Aparelho Locomotor, Universidade Federal de \\ Minas Gerais, Rua Engenheiro Albert Scharle, 30/701, Belo Horizonte, \\ MG, 30380370, Brasil (e-mail: danielbaumfeld@gmail.com).
}

\section{Resumo \\ Palavras-chave \\ - infecção dos ferimentos/ epidemiologia \\ - ferimentos por arma de fogo/ epidemiologia \\ - fraturas ósseas}

Objetivo Investigar a incidência de infecção em pacientes com fraturas por arma de fogo, e correlacionar esse achado com a ocorrência de desbridamento cirúrgico na sala de emergência.

Métodos Estudo retrospectivo, observacional e descritivo, que incluiu todos os casos de fraturas causadas por armas de fogo entre janeiro de 2010 e dezembro de 2014; foram incluídas 245 fraturas em 223 pacientes.

Resultados Houve infecção do local cirúrgico em 8,5\% das fraturas, e a média de desbridamentos necessários para controlar o processo infeccioso foi de 1,273 $\pm 0,608$. Foi identificada correlação entre o tratamento cirúrgico escolhido e o segmento corporal afetado $(p<0,001)$. O tratamento cirúrgico na sala de emergência teve correlação com a ocorrência de infecção ( $p<0,001$; teste do qui-quadrado).

Conclusão Pacientes com ferimentos à bala tratados de forma não operatória apresentaram lesões menos graves e estáveis; portanto, a incidência de complicações nesse grupo foi menor. Por outro lado, os pacientes com lesões complexas foram aqueles submetidos a desbridamento e fixação externa. Portanto, como esperado, foi encontrado um maior número de complicações infecciosas em pacientes submetidos à fixação externa.
Dados coletados no Hospital Risoleta Tolentino Neves, Universidade Federal de Minas Gerais, Belo Horizonte, Brasil. recebido

21 de Maio de 2019

aceito

29 de Novembro de 2019
DOI https://doi.org/

10.1055/s-0040-1702960. ISSN 0102-3616.
Copyright $\odot 2020$ by Sociedade Brasileira License terms de Ortopedia e Traumatologia. Published by Thieme Revinter Publicações Ltda, Rio de Janeiro, Brazil 


\section{Keywords}

- Wound infection/ epidemiology

- wounds, gunshot/ epidemiology

- fractures, bone and the affected body segment $(p<0.001)$. The surgical treatment in the emergency room had a correlation with the occurrence of infection ( $p<0.001$; Chi-squared test). Conclusion Patients with gunshot injuries treated non-operatively presented less severe and stable lesions; thus, the incidence of complications in this group was found to be lower. On the other hand, those patients with complex lesions underwent debridement and external fixation. Therefore, a greater number of infectious complications in patients submitted to external fixation was found, as expected.

\section{Introdução}

Lesões não fatais causadas por projéteis de arma de fogo (PAFs) afetam mais de 60 mil pessoas anualmente nos Estados Unidos (EUA), e as extremidades são as regiões anatômicas mais afetadas. ${ }^{1}$ Além disso, elas representam a segunda causa de morte nos EUA, e contabilizam-se entre 30 e 50 mil vítimas por ano. ${ }^{2}$

No Brasil, de acordo com o "Mapa da Violência 2015",3 estima-se que, entre 1980 e 2014, tenham ocorrido mais de 1 milhão de mortes secundárias a tiros: 8.710 em 1980, e $44.861 \mathrm{em} 2014$, o que representa um aumento de $415,1 \%$.

Ainda se debate sobre a melhor abordagem para o tratamento de fraturas causadas por PAFs. Alguns consideram que elas devam ser tratadas como fraturas expostas. Por outro lado, outros argumentam que nem todas as fraturas de PAFs requerem cirurgia e desbridamento urgentes. Taxas de infecção semelhantes foram identificadas em fraturas estáveis causadas por PAFs de baixa energia, com os pacientes recebendo ou não antibióticos profiláticos, por via oral ou intravenosa. ${ }^{4,5}$

Nosso principal objetivo foi o de investigar a incidência de infecção em pacientes com fraturas por arma de fogo, e correlacionar esse achado com a ocorrência de desbridamento cirúrgico na sala de emergência.

\section{Pacientes e Métodos}

Estudo retrospectivo, observacional, descritivo, que incluiu todos os casos de fraturas causadas por PAFs no Serviço de Ortopedia e Traumatologia do nosso hospital entre janeiro de 2010 e dezembro de 2014 . Os pacientes que morreram foram excluídos porque não havia registro de sua evolução, para infecção ou não, durante o acompanhamento ambulatorial.

As variáveis avaliadas foram: idade, segmento corporal acometido, tratamento de primeiros socorros, horário da sala de cirurgia, esquema antibiótico profilático, incidência de infecção do sítio cirúrgico, número de desbridamentos, e complicações.

A análise estatística foi realizada utilizando o aplicativo Wizard Pro para Mac, versão 1.9.24, e programa o StatPlus (StatPlus, Inc., Taipei, Taiwan), versão 6.7.03. As variáveis contínuas foram submetidas à avaliação da distribuição e posteriormente comparadas com o uso dos testes estatísticos apropriados. As variáveis categóricas foram comparadas pelo teste do qui-quadrado. Valores de $p<0,05$ foram utilizados para indicar diferença estatisticamente significante.

\section{Resultados}

Um total de 245 fraturas de 223 pacientes foram incluídas. Ao todo, 9 pacientes (4\%) com 10 fraturas foram excluídos do estudo porque não havia informações completas em seus prontuários.

A maioria dos pacientes era do sexo masculino (93,9\%; $n=230$ ), e a idade média foi de $26,0 \pm 1,2$ anos. Os membros superiores foram atingidos em $55,7 \%$ dos casos, os membros inferiores, em $43 \%$, e fraturas em múltiplos locais representaram $1,3 \%$ dos casos. A distribuição por segmento anatômico atingido é mostrada na - Tabela 1.

Quanto ao manejo médico na admissão, $28,1 \%$ dos pacientes não foram operados, e 71,9\% foram submetidos a procedimentos cirúrgicos, com a seguinte distribuição: $36,2 \%(n=85)$, apenas desbridamento; $24,3 \%(n=57)$, desbridamento e osteossíntese; 11,5\% $(n=27)$, desbridamento e fixação externa. No total, $95,9 \%$ dos procedimentos cirúrgicos foram realizados nas primeiras 24 horas após o trauma. 0 tempo médio de conversão entre fixador externo e osteossíntese foi de $14,37 \pm 2,7$ dias. Todos os pacientes incluídos receberam profilaxia com antibióticos durante o tempo médio de $2,779 \pm 0,214$ dias.

Houve infecção do local cirúrgico em $8,5 \%$ das fraturas, e a média de desbridamentos necessários para controlar o processo infeccioso foi $1,273 \pm 0,608$.

Foi identificada correlação entre o tratamento cirúrgico escolhido e o segmento corporal afetado $(p<0,001)$. Ao avaliar a conduta e sua relação com o segmento afetado, descobrimos que: 1) há preferência por não operar fraturas do úmero proximal e do quadril em comparação com fraturas

Tabela 1 Distribuição das fraturas por segmento anatômico

\begin{tabular}{|l|l|}
\hline Segmento & $\%(\mathbf{n})$ \\
\hline Perna & $17.9(42)$ \\
\hline Mão & $17(40)$ \\
\hline Antebraço & $15,3(36)$ \\
\hline Braço & $14(33)$ \\
\hline Coxa & $11,1(26)$ \\
\hline Ombro & $9,4(22)$ \\
\hline Pé & $8,5(20)$ \\
\hline Quadril & $5,5(13)$ \\
\hline Múltipla & $1,3(3)$ \\
\hline
\end{tabular}




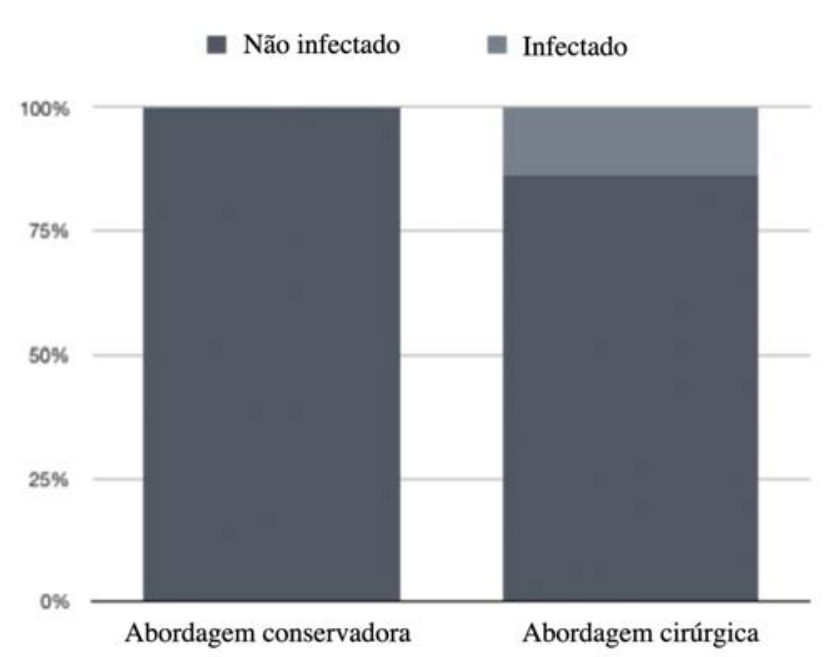

Fig. 1 Correlação entre infecção e conduta na sala de emergência (Teste do qui-quadrado; $p<0,001$ ).

de outros segmentos ( $p=0,004$ e $p<0,001$, respectivamente; teste do qui-quadrado); 2) o fixador externo não foi utilizado nas fraturas de quadril; e 3) todos os pacientes com fratura do pé foram operados.

O tratamento cirúrgico na sala de emergência teve correlação com a ocorrência de infecção ( $p<0,001$; teste do quiquadrado) (-Fig. 1).

A análise de subgrupos identificou que os pacientes submetidos a desbridamento e fixação externa foram responsáveis por $57,1 \%$ dos casos que evoluíram para infecção (escore Z, $p<0,001$ ) (-Fig. 2).

A proporção de complicações infecciosas foi maior entre os pacientes submetidos a desbridamento e fixação externa (teste do qui-quadrado; $p=0,04$ ).

O desbridamento externo e a fixação no pronto-socorro estiveram associados à ocorrência de complicações não infecciosas, como não união, consolidação viciosa e lesão neurológica (teste do qui-quadrado; $p=0,002$ ).

\section{Discussão}

Segundo a literatura, as lesões por PAFs são frequentes em homens $(94,4 \%)$ com idades entre 15 e 29 anos. $^{3}$ Os segmentos mais afetados são: coluna vertebral, fêmur, tíbia, fíbula, mão e antebraço. ${ }^{6,7}$ Nossos resultados são congruentes com os desses estudos: 93\% dos nossos pacientes eram do sexo masculino; a idade média foi de 26 anos; e a distribuição topográfica mostrou que os ossos longos foram os mais afetados. Este artigo é relevante para o estudo das complicações relacionadas às lesões por PAFs em uma população urbana brasileira.

O número de 245 fraturas em 223 pacientes é relevante, pois existem numerosos estudos ${ }^{8-10}$ retrospectivos sobre 0 assunto com amostra menor. Nguyen et al., ${ }^{8}$ em um estudo retrospectivo que avaliou os resultados do tratamento de fraturas articulares por PAFs, incluíram 55 lesões em 53 pacientes. Kaim Khani et al. ${ }^{9}$ avaliaram 90 lesões isoladas para comparar os resultados de lesões por PAFs de alta e baixa velocidade. Mehta et al. ${ }^{10}$ estudaram fraturas do antebraço causadas por PAFs por 5 anos, e incluíram 56 lesões de 55 pacientes. $O$ desenho retrospectivo do estudo justifica-se pela incidência de lesões por PAFs e pelo tempo necessário para a identificação das complicações relacionadas ao tratamento.

As lesões por PAFs são classificadas, de acordo com a velocidade do projétil, como baixas ou altas. Os ferimentos por PAFs de alta velocidade são causados por armas cujo projétil é lançado a velocidades superiores a $600 \mathrm{~m} / \mathrm{s}$. Os ferimentos de baixa velocidade são causados por revólveres comuns à população civil, enquanto os ferimentos de alta velocidade são típicos de armas militares. ${ }^{9}$ Neste estudo, supomos que as lesões estudadas foram causadas por armas com projéteis de baixa velocidade, uma vez que este é o armamento mais utilizado nos confrontos urbanos em nossa cidade. Vale ressaltar que neste estudo foram excluídos os pacientes que morreram, pois a avaliação do desfecho primário (infecção) exigiu o acompanhamento ambulatorial dos pacientes.

A gravidade das lesões que um PAF produz depende da energia transmitida aos tecidos, que, por sua vez, varia com a velocidade, diâmetro, forma, estabilidade na trajetória e peso do projétil. ${ }^{6} \mathrm{O}$ aumento do dano tecidual está associado a múltiplos PAFs, disparo próximo ao corpo, projéteis de alta velocidade, e munição com ponta oca. ${ }^{7}$ Neste artigo, devido ao seu caráter retrospectivo, não foi possível correlacionar as características do projétil ou as lesões produzidas com o resultado infeccioso. Podemos inferir que os pacientes não operados apresentaram lesões menos graves e mais estáveis no julgamento ortopédico; portanto, a incidência de complicações nesse grupo foi menor. Por outro lado, aqueles pacientes

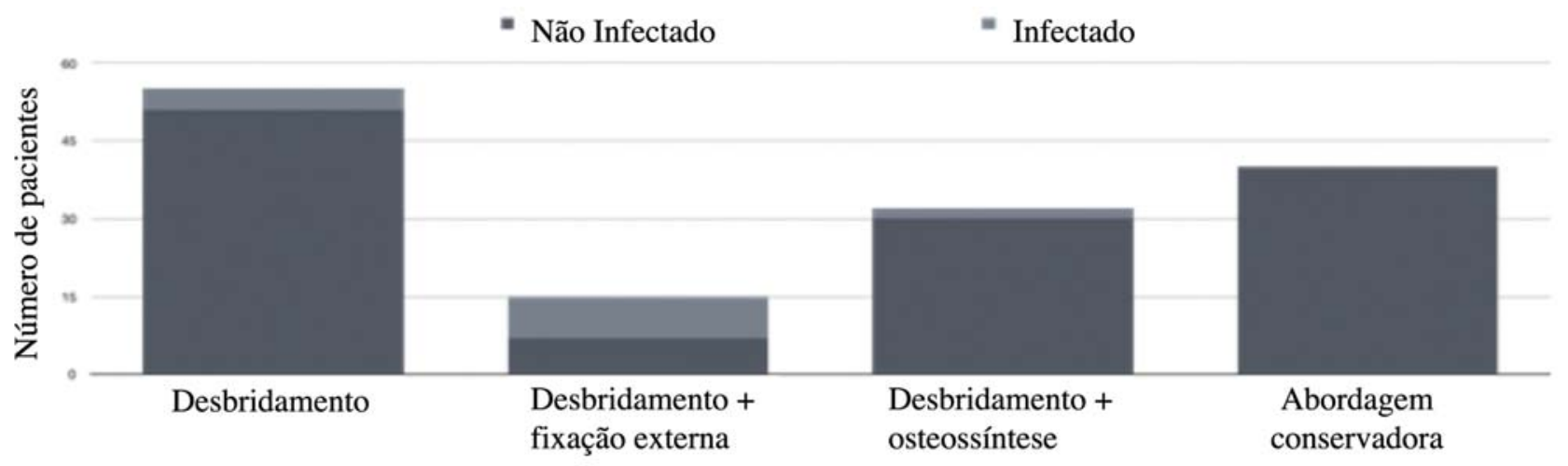

Fig. 2 Análise de subgrupo para identificar o grupo com maior incidência de infecção. 
com lesões complexas foram submetidos a desbridamento e fixação externa. Portanto, é explicado o maior número de complicações infecciosas em pacientes submetidos à fixação externa no primeiro atendimento.

As fraturas estáveis de baixa energia da tíbia e do úmero, por exemplo, podem ser adequadamente tratadas com talas ou moldadas com uma janela para tratamento de feridas e fechamento por segunda intenção. As fraturas instáveis são tratadas cirurgicamente com o mesmo princípio de estabilidade e implante que seria usado em fraturas fechadas. ${ }^{1}$

Nas fraturas causadas por PAF de alta ou baixa energia em ossos subcutâneos, como a tíbia ou a clavícula, recomenda-se o desbridamento cirúrgico da ferida. Quanto à profilaxia com antibióticos, nas fraturas causadas por PAFs de alta velocidade, são recomendadas de 24 a 72 horas de antibióticos intravenosos; nas fraturas causadas por PAFs de baixa velocidade, não há evidências para o uso de antibióticos. A escolha do regime antimicrobiano também é variável. ${ }^{7,11}$ Neste estudo, 40 pacientes (28\%) não foram submetidos a uma abordagem cirúrgica de emergência, e receberam antibióticos profiláticos por 24 horas. A decisão de não levar o paciente ao centro cirúrgico foi tomada pela equipe de plantão com base em uma avaliação pessoal da gravidade do caso. Essa estratégia, embora subjetiva, conseguiu garantir a esse grupo de pacientes uma menor incidência de complicações infecciosas e não infecciosas. Portanto, a gravidade da lesão inicial aparece como um fator para justificar uma maior incidência de complicações, apesar da abordagem inicial oferecida. A avaliação pela equipe de emergência também foi o critério utilizado para definir o tratamento em outros trabalhos. Nguyen et al. ${ }^{8}$ relataram que, em $43,6 \%$ dos casos, o tratamento não cirúrgico foi a opção, e não houve relatos de complicações infecciosas nesse grupo. No entanto, os dois casos de infecção relatados por esses autores são de pacientes submetidos a desbridamento cirúrgico de emergência, ${ }^{8,9}$ e eles correlacionaram a ocorrência de infecção profunda com a energia do trauma inicial. Na série destes autores, todos os 90 pacientes foram levados ao centro cirúrgico e receberam profilaxia com antibióticos. As lesões causadas por PAFs de alta energia apresentaram maior incidência de infecção, e as lesões múltiplas por PAFs foram excluídas. $^{9}$

Estudos recentes $^{8}$ não consideram que a classificação recomendada por Gustilo et al. ${ }^{4}$ deva ser usada na avaliação de fraturas causadas por PAFs. Os autores argumentam que 0 tamanho da lesão pós-desbridamento não corresponde ao dano causado internamente pelo PAF, e que a energia dissipada nos tecidos seria o principal fator prognóstico. ${ }^{6}$ Não utilizamos a classificação mencionada como parâmetro para estratificação da gravidade. Optamos por confiar no julga- mento da equipe de plantão sobre o grau de contaminação e instabilidade óssea, pois acreditamos que isso inclui outros elementos subjetivos que não podem ser simplificados pelas pontuações.

\section{Conclusão}

Pacientes com ferimentos a bala tratados de forma não operatória apresentaram lesões menos graves e estáveis; portanto, a incidência de complicações nesse grupo foi menor. Por outro lado, os pacientes com lesões complexas foram submetidos a desbridamento e fixação externa. Portanto, foi encontrado, conforme esperado, um maior número de complicações infecciosas em pacientes submetidos à fixação externa.

Conflito de Interesses

Os autores declaram não haver conflito de interesses.

\section{Referências}

1 Dougherty PJ, Vaidya R, Silverton CD, Bartlett CS III, Najibi S. Joint and long-bone gunshot injuries. Instr Course Lect 2010;59:465-479

2 Bartlett CS, Helfet DL, Hausman MR, Strauss E. Ballistics and gunshot wounds: effects on musculoskeletal tissues. J Am Acad Orthop Surg 2000;8(01):21-36

3 Waiselfisz JJ. Mapa Da Violência 2015: Mortes Matadas Por Armas de Fogo. Brasília, DF2015. Disponível em: httpbibjuventudeibict brjspuihandle

4 Dickey RL, Barnes BC, Kearns RJ, Tullos HS. Efficacy of antibiotics in low-velocity gunshot fractures. J Orthop Trauma 1989;3(01): 6-10

5 Knapp TP, Patzakis MJ, Lee J, Seipel PR, Abdollahi K, Reisch RB. Comparison of intravenous and oral antibiotic therapy in the treatment of fractures caused by low-velocity gunshots. A prospective, randomized study of infection rates. J Bone Joint Surg Am 1996;78(08):1167-1171

6 Moye-Elizalde GA, Ruiz-Martínez F, Suarez-Santamaría JJ, RuizRamírez M, Reyes-Gallardo A, Díaz-Apodaca BA. [Epidemiology of gunshot wounds at Ciudad Juárez, Chihuahua General Hospital]. Acta Ortop Mex 2013;27(04):221-235

7 Tosti R, Rehman S. Surgical management principles of gunshotrelated fractures. Orthop Clin North Am 2013;44(04):529-540

8 Nguyen MP, Reich MS, O'Donnell JA, et al. Infection and Complications After Low-velocity Intra-articular Gunshot Injuries. J Orthop Trauma 2017;31(06):330-333

9 Kaim Khani GM, Humail SM, Hafeez K, Ahmed N. Pattern of bony injuries among civilian gunshot victims at tertiary care hospital in Karachi, Pakistan. Chin J Traumatol 2015;18(03):161-163

10 Mehta SK, Dale WW, Dedwylder MD, Bergin PF, Spitler CA. Rates of neurovascular injury, compartment syndrome, and early infection in operatively treated civilian ballistic forearm fractures. Injury 2018;49(12):2244-2247

11 Simpson BM, Wilson RH, Grant RE. Antibiotic therapy in gunshot wound injuries. Clin Orthop Relat Res 2003;(408):82-85 